\title{
Ultrafast Demagnetization Excited by Extreme Ultraviolet Light From a Free-Electron Laser
}

André Philippi-Kobs ( $\square$ andre.kobs@desy.de )

Deutsche Elektronen-Synchrotron DESY https://orcid.org/0000-0001-7842-9577

Leonard Müller

Deutsches Elektronen-Synchrotron (DESY)

Magnus Berntsen

KTH Royal Institute of Technology

\section{Wojciech Roseker}

Deutsche Elektronen-Synchrotron DESY

Matthias Riepp

Deutsche Elektronen-Synchrotron DESY

Kai Bagschik

Deutsches Elektronen-Synchrotron DESY

Jochen Wagner

University of Hamburg

Robert Frömter

University of Hamburg

Miltcho Danailov

Elettra-Sincrotrone Trieste

Flavio Capotondi

Elettra Sincrotrone Trieste https://orcid.org/0000-0003-1980-6162

Emanuele Pedersoli

Elettra-Sincrotrone Trieste https://orcid.org/0000-0003-0572-6735

Michele Manfredda

Elettra-Sincrotrone Trieste

\section{Maya Kiskinova}

Elettra Sincrotrone Trieste https://orcid.org/0000-0001-9584-3526

Michal Stransky

Academy of Sciences of the Czech Republic

Vladimir Lipp

DESY https://orcid.org/0000-0002-5828-2296

Andreas Scherz

European X-Ray Free Electron Laser

Beata Ziaja 
Center for Free-Electron Laser Science CFEL, Deutsches Elektronen-Synchrotron DESY

\section{Hans Oepen}

Universität Hamburg

Gerhard Grübel

DESY

Article

Keywords: ultrafast demagnetization, extreme ultraviolet (XUV), free-electron lasers (FELs)

Posted Date: October 8th, 2021

DOl: https://doi.org/10.21203/rs.3.rs-955056/v1

License: (c) (1) This work is licensed under a Creative Commons Attribution 4.0 International License. Read Full License 


\title{
Ultrafast Demagnetization Excited by Extreme Ultraviolet Light From a Free-Electron Laser
}

\author{
André Philippi-Kobs ${ }^{1,2} \rrbracket$, Leonard Müller ${ }^{1,3}$, Magnus H. Berntsen ${ }^{1,4}$, Wojciech Roseker ${ }^{1}$, \\ Matthias Riepp", Kai Bagschik ${ }^{1,2}$, Jochen Wagner², Robert Frömter², Miltcho Danailov ${ }^{5}$, Flavio \\ Capotondi $^{5}$, Emanuele Pedersoli ${ }^{5}$, Michele Manfredda $^{5}$, Maya Kiskinova ${ }^{5}$, Michal Stransky ${ }^{6}$, \\ Vladimir Lipp ${ }^{7}$, Andreas Scherz ${ }^{8}$, Beata Ziaja ${ }^{7,9}$, Hans Peter Oepen ${ }^{2}$, Gerhard Grübel ${ }^{1}$
}

Ultrashort and intense extreme ultraviolet (XUV) and X-ray pulses readily available at freeelectron lasers (FELs) enable studying non-linear light-matter interactions on femtosecond timescales. Here, we report on the non-linear fluence dependence of magnetic scattering of Co/Pt multilayers, using FERMI FEL's 70-fs-long single and double XUV pulses, the latter with a temporal separation of $200 \mathrm{fs}$, with a photon energy slightly detuned to the Co $M_{2,3}$ absorption edge. We observe a quenching in magnetic scattering that sets-in already in the nondestructive fluence regime of a few $\mathrm{mJ} / \mathrm{cm}^{2}$ typically used for FEL-probe experiments on magnetic materials. Calculations of the transient electronic structure in tandem with a phenomenological modeling of the experimental data by means of ultrafast demagnetization unambiguously show that XUV-radiation-induced demagnetization is the dominant mechanism for the quenching in the investigated fluence regime of $<50 \mathrm{~mJ} / \mathrm{cm}^{2}$, while light-induced changes of the electronic core levels are predicted to additionally occur at higher fluences. The modeling of the data further indicates that the demagnetization proceeds on the sub-20-fs timescale. This ultrashort timescale is consistent with non-coherent models for ultrafast demagnetization, considering the sub-femtosecond lifetime of hot electrons with energies of a few $10 \mathrm{eV}$ generated by the XUV radiation.

\footnotetext{
1Deutsches Elektronen-Synchrotron DESY, Notkestraße 85, 22607 Hamburg, Germany. ${ }^{2}$ Institut für Nanostruktur- und Festkörperphysik, Universität Hamburg, Luruper Chaussee 149, 22761 Hamburg, Germany. ${ }^{3}$ Fachbereich Physik, Universität Hamburg, Jungiusstraße 9, 20355 Hamburg, Germany. ${ }^{4}$ Department of Applied Physics, KTH Royal Institute of Technology, AlbaNova Universitetscentrum, 10691 Stockholm, Sweden. ${ }^{5}$ Elettra-Sincrotrone Trieste, Strada Statale 14-km 163.5, 34149 Basovizza, Trieste, Italy. ${ }^{6}$ Department of Radiation and Chemical Physics, Academy of Sciences of the Czech Republic, Na Slovance 2, 18221 Prague, Czech Republic. ${ }^{7}$ Center for Free-Electron Laser Science, DESY, Notkestraße 85, 22607 Hamburg, Germany. ${ }^{8}$ European XFEL GmbH, Holzkoppel 4, 22869 Schenefeld, Germany. ${ }^{9}$ Institute of Nuclear Physics, Polish Academy of Sciences, Radzikowskiego 152, 31-342 Krakow, Poland.

Ðemail: andre.kobs@desy.de; mhbe@kth.se
} 
Free-electron lasers (FELs) enable studies on dynamics of matter on combined femtosecondtime and nanometer-length scales [1,2]. One intriguing topic within contemporary research on magnetism - ultrafast near-infrared (NIR) laser-induced demagnetization [3-6] - benefits greatly from FELs since the transient response of the atomic lattice [7] and nanoscale magnetic heterogeneities can be studied with chemical sensitivity [8-11]. Further, the high photon densities provided by FELs can generate in matter ultrafast, non-linear responses and highlyexcited transient states $[1,2,12-19]$. Studies conducted recently tackle the non-linear intrapulse response of magnetic materials upon FEL radiation [19-23], thereby expanding the study on ultrafast magnetization dynamics beyond the regime of NIR radiation. These studies rely on resonant techniques making use of the X-ray magnetic circular dichroism (XMCD) effect that is particularly sensitive to the magnetization and any further change in population and energy levels of the involved electronic states.

The starting point of the investigation of the nonlinear interaction of ultra-short FEL radiation and magnetic materials was the observation of a drastic quenching of the magnetic scattering signal of Co/Pt multilayers within the sub-100-fs long pulse duration, when using XUV-pulses with a fluence of $f=5 \mathrm{~J} / \mathrm{cm}^{2}$, resonantly tuned to the Co $M_{2,3}$ edge at $E_{\mathrm{ph}}=59.6 \mathrm{eV}[20]$. This quenching was explained by a shift of the $3 p$ core levels to lower energies due to the charged environment. At higher photon energies, corresponding to the Co $L_{3}$ edge $\left(E_{\mathrm{ph}}=778 \mathrm{eV}\right)$, a quenching of the magnetic-scattering signal was also observed for fluences $f \gtrsim$ $10 \mathrm{~mJ} / \mathrm{cm}^{2}$ [21] and was assigned to stimulated emission [22,23]. In the hard X-ray regime, magnetic-scattering experiments with high fluences are not reported [24]. However, Yoneda et al. performed X-ray-emission spectroscopy experiments for $\mathrm{Fe} \mathrm{[19]} \mathrm{and} \mathrm{Cu}$ [18] using radiation resonantly tuned to the $K$ edges. They found a saturable absorption accompanied by a band shift [19] and stimulated emission [18] for fluences $f \gtrsim 10^{5} \mathrm{~J} / \mathrm{cm}^{2}$. Recently, ultrafast demagnetization was additionally put forward to explain the quenching of magnetic scattering [25] and XMCD effect in absorption [26] for FEL radiation resonantly tuned to the Co $M_{2,3}$ and $L$ edge, respectively [27]. In light of the contrasting explanations for the non-linear photonmagnetic material interaction, it becomes clear that a variety of mechanisms may play a role in the quenching of XMCD effect-based signals.

Here, we report on the XUV-fluence dependence of the resonant magnetic small-angle X-ray scattering (mSAXS) of Co/Pt multilayers. For the experiments, single and double-FEL-pulse modes with photon energies tuned closely above the $M_{2,3}$ edge of Co are used (Fig. 1(a)), enabling an unambiguous determination whether band shift, stimulated emission or demagnetization is the dominant mechanism. For both FEL modes, a quenching of magnetic scattering with increasing fluence is observed. Both sets of data are well-described by a phenomenological model that was developed to describe XUV-radiation-induced 
demagnetization, while calculations on light-induced transient changes in the population and energy level of the $3 p$ shell cannot explain the experimental data. Our study provides evidence for the existence of ultrafast demagnetization in the XUV regime and additionally indicates that the demagnetization proceeds on a sub-20-fs timescale. This result is supported by the extremely short lifetime of XUV-generated hot electrons even when only considering conventional, i.e., non-coherent, demagnetization mechanisms, as detailed for Elliott-Yafet spin-flip scattering [4].

\section{Results}

Magnetic small-angle X-ray scattering and scattering efficiency. The mSAXS experiments on a $(0.8 \mathrm{~nm} \mathrm{Co} / 1.4 \mathrm{~nm} \mathrm{Pt})_{16}$ multilayer, which exhibits perpendicular magnetic anisotropy [29] (see Methods) and nanoscale magnetic domains [9], were performed utilizing 70-fs-long circularly-polarized XUV-pulses at FERMI's DiProl beamline [30]. Two different FEL operation modes were used, namely the conventional single-pulse (1P) as well as the double-pulse mode (2P) [31,32] with a pulse separation of $200 \mathrm{fs}$ (Fig. 1(a), Methods). The wavelength of the FELradiation for the single-pulse mode was $\lambda_{\mathrm{ph}, 1 \mathrm{P}}=20.3 \mathrm{~nm}\left(E_{\mathrm{ph}, 1 \mathrm{P}}=61.1 \mathrm{eV}\right)$, whereas in the double-pulse mode the wavelengths of the sub-pulses were shifted by $\Delta \lambda \approx \pm 0.035 \mathrm{~nm}$ with respect to $\lambda_{\text {ph,1P }}$ (Fig. 1(b)) allowing for a spectroscopic detection of the sub-pulse intensities (Fig. 1(b), inset). All wavelengths were blue shifted with respect to the maximum in magnetic-scattering cross section located at $\lambda_{\mathrm{ph}, \mathrm{Co}-M_{2,3} \text { edge }}=20.8 \mathrm{~nm}\left(E_{\mathrm{Co}-M_{2,3} \text { edge }}=59.6 \mathrm{eV}\right.$; Fig. 1(b)) [33]. The blue shift ensures a comparable scattering cross section for all used wavelengths, allowing for a direct comparison of the single and double-pulse data. Furthermore, it enables identifying the impact of the electronic level shift on the quenching as outlined below.

The radial intensity $S$ vs scattering vector $Q$ is obtained by azimuthal averaging of the scattered intensity (Fig. 1(c)). The peak position $Q_{\text {peak }} \approx 0.036 \mathrm{~nm}^{-1}$ corresponds to an average domain width of $\approx 90 \mathrm{~nm}$, consistent with previous studies on a nominally identical sample [8]. The peak value $S\left(Q_{\text {peak }}\right)$ serves as a quantitative measure for the strength of the magneticscattering cross section (Methods), given by [34]

$S\left(Q_{\text {peak }}\right) \propto\left|G_{1}\right|^{2} M_{\mathrm{S}}^{2}$ with $G_{1}=\frac{\left|<3 d, m_{l}\right| C_{-1}^{(1)}\left|3 p, m_{j}>\right|^{2}-\left|<3 d, m_{l}\right| C_{+1}^{(1)}\left|3 p, m_{j}>\right|^{2}}{E_{\mathrm{ph}}-E_{\text {edge }}+i(\Delta / 2)}$.

$M_{\mathrm{S}}$ is the saturation magnetization, $\left|<3 d, m_{l}\right| C_{ \pm 1}^{(1)}\left|3 p, m_{j}>\right|^{2}$ the dipole-transition matrixelement describing core-to-valence transitions between $3 p$ and $3 d$ states under the action of the dipole-operator $C_{ \pm 1}^{(1)}$, and $\Delta$ the resonance width of the $3 d$ states [34].

As a measure for the quenching, we define the scattering efficiency $I_{\text {eff }}$ as the ratio of peak intensity $S\left(Q_{\text {peak }}\right)$ and pulse energy $E_{\text {pulse }}$ (proportional to the number of incident photons) 
normalized to one at small fluences $f<1 \frac{\mathrm{mJ}}{\mathrm{cm}^{2}}$. The scattering efficiency $I_{\text {eff }}$ vs peak fluence $f$ behavior, extracted from single-pulse and double-pulse experiments, is given in Fig. 2. For the latter, $f$ is the sum of the peak-fluences of both sub-pulses, hence for the same $f$, the single pulse has twice the peak-intensity as compared to the sub-pulses in the double-pulse mode. Both $I_{\text {eff }}(f)$ datasets can be described by single exponentials $\left(I_{\text {eff }}(f)=\exp \left(-\frac{f}{\xi}\right)\right)$ suggesting the presence of one dominant mechanism for quenching. Characteristic fluences of $\xi_{1 \mathrm{P} \text {,exp }}=$ $(26.3 \pm 1.1) \mathrm{mJ} / \mathrm{cm}^{2}$ and $\xi_{2 \mathrm{P}, \text { exp }}=(25.1 \pm 0.8) \mathrm{mJ} / \mathrm{cm}^{2}$ are obtained, reflecting the similarity of both curves within the experimental resolution. As shown below, this similarity is a fingerprint for ultrafast demagnetization occurring on a sub-20-fs timescale. Note that the quenching sets in already in the few- $\mathrm{mJ} / \mathrm{cm}^{2}$ regime, which is much lower than the observed damage threshold of $f_{\text {damage }} \approx 20 \mathrm{~mJ} / \mathrm{cm}^{2}$, i.e., the minimum fluence irreversibly altering the sample's scattering pattern.

Modeling. In order to determine the underlying mechanism for quenching, the experimental results are compared with different models (for details see Supplement A-C). Calculations on the light-matter interaction using kinetic Boltzmann equations [35] (Supplement B) show the expected low-fluence behavior (linear-response regime), namely the $3 p-3 d$ absorption process (Fig. 3(a)) is dominant and the preponderant decay-channel is the Auger process (Fig. 3(b)). Around $88 \%$ of the Auger processes with a sub-femtosecond decay time of $\tau_{\text {Auger }}=0.4 \mathrm{fs}$ are $3 d-3 p$ transitions refilling the $3 p$ shell combined with excitations of $3 d$ electrons up to an energy of $60 \mathrm{eV}$ above the $3 d$ level. Since $\tau_{\text {Auger }}$ is two orders of magnitude shorter than the pulse length, significant intra-pulse repopulation of the $3 p$ shell occurs.

Fig. 3 further illustrates the impact of stimulated emission (c), p-level shift (d), and ultrafast demagnetization (e) on the electronic structure in the respective high-fluence regimes together with the corresponding calculated $I_{\text {eff }}(f)$ behaviors (f). As detailed in the supplement (section $A$ and $B$ ) and visualized in Fig. 3(f), the results of modeling show that the experimentally observed quenching of magnetic scattering cannot, neither qualitatively nor quantitatively, be a consequence of a significant amount of excited-state population, or a $p$-level shift modifying $G_{1}$ in Eq. (1). In contrast to the experimental results, for the stimulated emission scenario the double-pulse $I_{\text {eff }}(f)$ curve is shifted to twice the fluence as compared to the single-pulse $I_{\text {eff }}(f)$ curve (dashed curves in Fig. 3(f)) since the (peak) intensity dictates the amount of excited-state population. Also in contrast to the experimental curve, $I_{\text {eff }}(f)$ initially increases for a $p$-shell-shift induced by a charged environment of excited electrons (dotted curves). The latter is a consequence of the experimentally used blue shift of the photon energy with respect 
to the unperturbed $3 p-3 d$ transition and the fact that with increasing fluence the resonance energy of the $3 p-3 d$ transition gradually shifts to higher energies.

A plausible scenario to explain the quenching is an XUV-induced reduction of saturation magnetization $M_{\mathrm{S}} \propto \sqrt{S\left(Q_{\text {peak }}\right)}$ (Eq. (1)) as it provides an explanation for the observed shift of the double-pulse $I_{\text {eff }}(f)$ curve with respect to the single-pulse $I_{\text {eff }}(f)$ curve towards smaller fluences (solid lines) as outlined in the following.

Fig. 4(a) and the solid lines in Fig. 3(f) show the impact of demagnetization on $I_{\text {eff }}(f)$ deduced from a phenomenological model (Supplement $\mathrm{C}$ ) for a pulse length of $70 \mathrm{fs}$ and a delay time of $200 \mathrm{fs}$ resembling the experimental parameters. For the modeling, based on experimental observations for demagnetization with NIR light, an exponential decay of $M_{S}$ in time is assumed whose strength scales linearly with fluence $[9,36,37]$. Remagnetization is neglected since it typically occurs on picosecond timescales. Hence, photons impinging on the sample at time $t^{\prime}$ initiate demagnetization (proceeding for $t>t^{\prime}$ ) with a characteristic time $\tau_{\text {demag }}$ and strength proportional to the instantaneous intensity $I\left(t^{\prime}\right)$ leading to a relative reduction of the saturation magnetization $M_{\mathrm{S}}$ according to $\delta M_{\mathrm{S}}\left(t, t^{\prime}\right) \propto I\left(t^{\prime}\right) \cdot\left(1-\exp \left(-\frac{t-t^{\prime}}{\tau_{\mathrm{demag}}}\right)\right)$. The transient $M_{\mathrm{S}}$ normalized to one is then given by $M_{\mathrm{S}}(t)=1-\int \delta M_{\mathrm{S}, k}\left(t, t^{\prime}\right) d t^{\prime}$, which is shown in Fig. 4(b) for three selected fluences for single and double-pulse mode. The transient magnetic-scattering intensity is proportional to $M_{S}^{2}(t) \cdot I(t)$, and the integration over time $t$, normalized to the fluence $f$, finally provides $I_{\text {eff }}(f)$ (Supplement $\mathrm{C}$ ). As a result, since $M_{\mathrm{S}}(t)$ monotonically decreases (Fig. 4(b)), a stronger reduction of the scattering intensity occurs during the second sub-pulse, and hence, opposite to stimulated emission, the double-pulse $I_{\text {eff }}(f)$ curve is shifted towards smaller fluences with respect to the single-pulse $I_{\text {eff }}(f)$ curve (Fig. 3(f) and Fig. 4(a)). Besides pulse length and delay time, the shift depends on the demagnetization time. For the NIRdemagnetization time of $\mathrm{Co}, \tau_{\text {demag,NIR,Co }} \approx 200 \mathrm{fs}$ [4,38-40], resembling the used delay time between the sub-pulses, the modeling gives a shift by a factor of $\xi_{1 \mathrm{P}} / \xi_{2 \mathrm{P}} \approx 2.75$. Compared to thick elemental films, for alloys and multilayers containing high- $Z$ material ( $Z$ : atomic number) like $\mathrm{Pt}$, a significantly shorter demagnetization time is generally found [40,41], indicating an important impact of spin-orbit coupling on ultrafast demagnetization according to a $\tau_{\text {demag }} \propto$ $1 /\left(Z^{4} T_{\mathrm{C}}\right)$ scaling [40]. Note that particularly for multilayers, both the Curie temperature $T_{\mathrm{C}}$ and the effective $Z$ depends on the detailed morphology of the interfaces. The shorter $\tau_{\text {demag }}$ the smaller is the shift between the single and double-pulse curves, e.g., for $\tau_{\mathrm{demag}, \mathrm{NIR}, \mathrm{Co} / \mathrm{Pt}}=40 \mathrm{fs}$ observed as the shortest $\tau_{\text {demag }}$ for $\mathrm{Co} / \mathrm{Pt}$ [40], the shift obtained from the model reduces to $\xi_{1 \mathrm{P}} / \xi_{2 \mathrm{P}} \approx 1.5$. In the extreme case, for $\tau_{\text {demag }}$ significantly shorter than the pulse length, the 
single-pulse and double-pulse curves converge. Hence, the experimental ratio of $\xi_{1 \mathrm{P}, \exp } / \xi_{2 \mathrm{P}, \exp } \approx 1.05$ indicates a relatively short demagnetization time.

In order to determine the demagnetization time, the phenomenological model for demagnetization is simultaneously fitted to the single and double-pulse data (Fig. 2). An upper bound for the demagnetization time of $\tau_{\text {demag,XUV,Co/Pt }} \leq 20 \mathrm{fs}$ is obtained which is at least a factor of two shorter compared to $\tau_{\mathrm{demag,NIR,Co/Pt}}[40]$. Recently, a slight reduction of the demagnetization time for using XUV compared to NIR radiation was also reported for Co/Pd [25].

\section{Discussion}

How can we understand a sub-20-fs demagnetization time? Very recently, comparably fast demagnetization times down to the sub-20 fs regime were reported even for NIR laser-induced demagnetization in $\mathrm{Co} / \mathrm{Cu}$ [42] and Ni/Pt [43] and attributed to spin transfer across chemical interfaces. Both studies indicate that three different processes promote demagnetization occurring on subsequent time scales, namely coherent spin transfer and back transfer from ferromagnetic to paramagnetic lattice sites for $t<20 \mathrm{fs}$, referred to as optically induced coherent spin transfer (OISTR) in Ref. [43], followed by coherent OISTR+SOC-mediated spin flips for $20 \mathrm{fs}<t<100 \mathrm{fs}$. Finally, for timescales $t>100 \mathrm{fs}$ conventional, non-coherent demagnetization processes as local Elliott-Yafet-based scattering processes [44] and/or nonlocal superdiffusive spin currents occur [45].

Hereafter, the XUV radiation-induced demagnetization process is discussed with emphasis on explaining the sub-20-fs demagnetization time using conventional, i.e., non-coherent, demagnetization mechanisms. About $2 / 3$ of the XUV-induced excitations are inter-band $3 p-3 d$ dipole transitions [33] followed by an Auger decay leading to hot electrons with energies of up to $60 \mathrm{eV}$ above the Fermi level. The resulting fraction of photo-ionized Co is only $\approx 20 \%$ for the maximum fluence used experimentally, $f=43 \mathrm{~mJ} / \mathrm{cm}^{2}$ (Supplement $\mathrm{B}$ ), so that even for a possibly high polarization of the Auger electrons of a few $10 \%$ [46-47], the disparity of $3 d^{\uparrow}$ and $3 d^{\downarrow}$ electrons, i.e., $M_{\mathrm{S}}$, is not significantly affected by the Auger processes. Consequently, subsequent electron-scattering processes trigger demagnetization analogous to conventional NIR-light-induced demagnetization, however, with a significantly shorter demagnetization time. In the following it is shown that the key for a faster demagnetization are the fast electron scattering processes upon XUV-radiation. As the electron-electron scattering with a characteristic lifetime $\tau_{\mathrm{e}^{-}} \mathrm{e}^{-}$is the predominant scattering process for $60-\mathrm{eV}$-hot electrons [48], it is assumed that the demagnetization is mediated by the Elliott-Yafet mechanism based on electron-electron Coulomb-scattering in the presence of spin-orbit interaction [49]. According to the Elliott-Yafet relation, the spin-relaxation time $\tau_{\uparrow \downarrow}$ is proportional to the relaxation time $\tau$ 
of the electrical resistivity ( $\tau \approx \tau_{\mathrm{e}^{-} \mathrm{e}^{-}}$for hot electrons): $\frac{1}{\tau_{\uparrow \downarrow}}=a_{\mathrm{sf}} \frac{1}{\tau_{\mathrm{e}^{-} \mathrm{e}^{-}}}$[50], where $a_{\mathrm{sf}}$ is the spinflip probability of the scattering processes [4,50]. As $\tau_{\mathrm{e}^{-}} \mathrm{e}^{-}$of high-energy electrons is short, a faster demagnetization for $60-\mathrm{eV}$-hot electrons can be expected. According to Fermi's golden rule the scaling-relation $\tau_{\mathrm{e}^{-} \mathrm{e}^{-}} \propto\left(E_{\mathrm{e}^{-}}-E_{\mathrm{F}}\right)^{-2}$ is applicable for low-energy electrons of a few $\mathrm{eV}$ [48]. For a wider energy range $\left(0 \leq E_{\mathrm{e}^{-}} \leq 10 \mathrm{keV}\right)$, the phenomenological description of the universal inelastic mean free path vs electron-energy curve is better suited to estimate $\tau_{\mathrm{e}^{-} \mathrm{e}^{-}}$[51] (Supplement E). As a result $\tau_{\mathrm{e}^{-} \mathrm{e}^{-}}(60 \mathrm{eV}) \approx 77$ as and $\tau_{\mathrm{e}^{-} \mathrm{e}^{-}}(1.5 \mathrm{eV}) \approx 71 \mathrm{fs}$ are

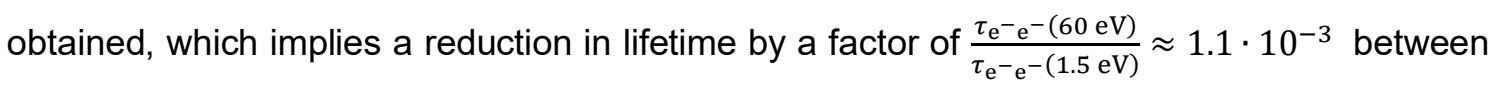
1.5-eV and $60-\mathrm{eV}$-hot electrons. These $\tau_{\mathrm{e}^{-}} \mathrm{e}^{-- \text {values }}$ are systematically larger than the experimental results for ferromagnets. For Co $\tau_{\mathrm{e}^{-}} \mathrm{e}^{-}(1.2 \mathrm{eV}) \approx 6 \mathrm{fs}$ was experimentally determined, i.e., one order of magnitude shorter than for $\mathrm{Ag}$, which was explained by the high density of states near $E_{\mathrm{F}}$ in Co [52,53]. Recently, the XUV-excited electron lifetime in Ni was found to be in the 10 as range, i.e., $\approx 100$ as shorter as compared to $\mathrm{Cu}$, which was explained in terms of spin-dependent scattering in $\mathrm{Ni}$ [54]. Hence, assuming a similarly shorter $\tau_{\mathrm{e}^{-} \mathrm{e}^{-}}(60 \mathrm{eV})$ for $\mathrm{Co}$, the above-mentioned $\frac{\tau_{\mathrm{e}^{-}} \mathrm{e}^{-(60 \mathrm{eV})}}{\tau_{\mathrm{e}^{-} \mathrm{e}^{-}(1.5 \mathrm{eV})}}$ value remains plausible. Assuming $\tau_{\uparrow \downarrow}(1.5 \mathrm{eV})=200 \mathrm{fs}$ and a spin-flip probability $a_{\mathrm{sf}}$ independent of electron-energy results in a spin-relaxation time in the sub-femtosecond regime of $\tau_{\uparrow \downarrow}(60 \mathrm{eV})=\tau_{\uparrow \downarrow}(1.5 \mathrm{eV}) \frac{\tau_{\mathrm{e}} \mathrm{e}^{-}(60 \mathrm{eV})}{\tau_{\mathrm{e}^{-}} \mathrm{e}^{-(1.5 \mathrm{eV})}} \approx$ 0.2 fs. Since $\tau_{\uparrow \downarrow}(60 \mathrm{eV}) \approx \tau_{\text {Auger }}(60 \mathrm{eV})$, the demagnetization time in the XUV-regime is determined by both, Auger-decay time (principally acting as a delay-time) and spin-relaxation time, i.e., $\tau_{\text {demag }}=\tau_{\text {Auger }}+\tau_{\uparrow \downarrow} \lesssim 1 \mathrm{fs}$.

The description of the faster demagnetization in the XUV regime in terms of electron-electron scattering should not rule-out other probable processes, proposed to explain ultrafast demagnetization. For instance, electron-phonon-mediated spin-flip-scattering processes might be much faster for an excitation with $60-\mathrm{eV}$ than for $1.5-\mathrm{eV}$ photons. In fact, the electronphonon interaction is reported to increase with the energy of the electronic system [45,55-57], such that, in line with above reasoning, faster demagnetization is plausible. Future quantitative descriptions have to include the role of the Pt layers, as they significantly increase the spinorbit interaction of $\mathrm{Co}$ at the Co/Pt interfaces [40], play a decisive role for the coherent OISTR effect [43], and provide a spin sink for superdiffusive spin-polarized currents along the film normal [45]. Since the velocity of 60-eV-hot electrons is in the order of a few $\mathrm{nm} / \mathrm{fs}$ [51] a fast spin transport from the $0.8 \mathrm{~nm}$ thick Co to the Pt layers is reasonable providing an alternative conventional (non-coherent) explanation for a sub-20-fs demagnetization time.

The fitting of the experimental data using the model for demagnetization also provides the fluence $f_{\text {demag,Xuv }}$ leading to complete demagnetization (Fig. 2) when additionally considering 
the impact of the two-dimensional Gaussian-beam profile, as outlined in Supplement C and D. Remarkably, $f_{\text {demag,Xuv }}=(12 \pm 3) \mathrm{mJ} / \mathrm{cm}^{2}$ is similar to the value estimated for the same multilayer using NIR radiation $\left(E_{\mathrm{ph}}=1.5 \mathrm{eV}: f_{\text {demag,NIR }} \approx 18 \mathrm{~mJ} / \mathrm{cm}^{2}\right)$ [8]. In fact, considering the slightly different attenuation lengths for $\mathrm{Co}, \ell_{\mathrm{att}, \mathrm{Co}}$, for both photon energies $\left(\ell_{\mathrm{att}, \mathrm{Co}}(60 \mathrm{eV}) \approx 9 \mathrm{~nm}\right.$ [33], $\ell_{\mathrm{att}, \mathrm{Co}}(1.5 \mathrm{eV}) \approx 13 \mathrm{~nm}$ [58]), a similar fluence is absorbed by the Co layers for both $f_{\text {demag }}$ values. Since the demagnetization was found to scale with the absorbed fluence, independent of the optical wavelength $[59,60]$, the estimated $f_{\text {demag,XuV }}$ value appears reasonable under the prerequisite that the same mechanisms dictate ultrafast demagnetization for both regions of the electromagnetic spectrum. In contrast, a more than one order of magnitude higher $f_{\text {demag,Xuv }}$ was reported recently for XUV-FEL radiation with similar pulse characteristics (spectrum, pulse length, coherence) [25] for which stimulated emission and a $3 p$-band shift are expected to significantly affect the magnetic scattering signal (Fig. 3(f)).

In conclusion, the quenching of magnetic scattering as a function of fluence was investigated, using 70-fs-long single and double-pulse XUV FEL radiation. The quenching sets in for nondestructive XUV fluences in the $\mathrm{mJ} / \mathrm{cm}^{2}$ range, defining a photon-flux limitation for FEL-probe experiments on magnetic systems. The similarity of single and double-pulse data shows that the quenching occurs on a very short sub-20 fs timescale. The driving mechanism for the quenching effect is identified to be XUV-induced demagnetization. The sub-20 fs timescale for demagnetization can be understood in a conventional manner considering the extremely-short lifetime of few-10-eV-hot electrons. Stimulated emission and transient shifts of the $3 p$ level are predicted to occur at higher fluences in the $>50 \mathrm{~mJ} / \mathrm{cm}^{2}$ regime. By tailoring the photon-pulse properties, future investigations need to identify the ultimate timescale for light-induced coherent and non-coherent manipulation of magnetization [43], i.e., tackling the question, if demagnetization can proceed even on the sub-femtosecond timescale. 


\section{Methods}

Magnetic Sample. As sample system, a Co/Pt multilayer with perpendicular magnetic anisotropy grown by sputtering techniques on $50 \mathrm{~nm}$ thick $\mathrm{Si}_{3} \mathrm{~N}_{4}$ membranes with lateral dimensions of $200 \times 200 \mu \mathrm{m}^{2}$ was used [29]. The thicknesses of the individual Co and Pt layers were $0.8 \mathrm{~nm}$ and $1.4 \mathrm{~nm}$, respectively, and a total of 16 repetitions of the $\mathrm{Co}$ and $\mathrm{Pt}$ was deposited on a $\mathrm{Pt}$ seed layer of $5 \mathrm{~nm}$ thickness, i.e., $\mathrm{Pt} /(5.0 \mathrm{~nm}) /[\mathrm{Co}(0.8 \mathrm{~nm}) / \mathrm{Pt}(1.4 \mathrm{~nm})] 16 / \mathrm{Pt}(0.6 \mathrm{~nm})$. The $\mathrm{Pt}$ cap layer is $2 \mathrm{~nm}$ thick to prevent oxidation. After growth, the sample was demagnetized in an out-of-plane magnetic field to generate a labyrinthlike close-to-equilibrium domain state with an average domain size of $\approx 90 \mathrm{~nm}$. A chip with a total of 400 membranes (array of $20 \times 20$ with a pitch in both directions of $1 \mathrm{~mm}$ ) was used for the experiment in order to enable repeated and comparable destructive single-shot measurements on membranes from the same production batch.

Scattering mechanism. The peak value of the distribution function $S\left(Q_{\text {peak }}\right)$ serves as a quantitative measure for the strength of the scattering cross section $\sigma=f^{2}$, where $f$ is the scattering factor $f\left(\mathbf{Q}, E_{\mathrm{ph}}, \boldsymbol{\varepsilon}\right)=f^{0}(\mathbf{Q})+f^{\prime}\left(E_{\mathrm{ph}}, \boldsymbol{\varepsilon}, \mathbf{M}\right)-i f^{\prime \prime}\left(E_{\mathrm{ph}}, \boldsymbol{\varepsilon}, \mathbf{M}\right)[34]$. Here, $f^{0}$ is the atomic form factor corresponding to the atomic number $Z$ for forward scattering, while $f^{\prime}$ and $f^{\prime \prime}$ are the anomalous scattering factors. $\boldsymbol{\varepsilon}$ is the light's polarization unit vector and $\mathbf{M}$ the magnetization. As no charge inhomogeneity exists on the length scale of the magnetic multi-domain pattern, i.e., $100 \mathrm{~nm}$, the azimuthally averaged $S(Q)$ (including the peak value $S\left(Q_{\text {peak }}\right)$ ) are only sensitive to the magnetic part of the scattering factor [61], which for light propagation parallel/antiparallel to $\mathbf{M}$ is given by [34]

$S\left(Q_{\text {peak }}\right) \propto\left|G_{1}\left(\boldsymbol{\varepsilon}_{\text {final }}^{*} \times \boldsymbol{\varepsilon}_{\text {initial }}\right) \cdot \mathbf{M}\right|^{2}=\left|G_{1}\right|^{2} M_{\mathrm{S}}^{2} \quad$ with $G_{1}=\frac{\left|<3 d, m_{l}\right| C_{-1}^{(1)}\left|3 p, m_{j}>\right|^{2}-\left|<3 d, m_{l}\right| C_{+1}^{(1)}\left|3 p, m_{j}>\right|^{2}}{E_{\mathrm{ph}}-E_{\text {edge }}+i(\Delta / 2)}$.

$\left|<3 d, m_{l}\right| C_{ \pm 1}^{(1)}\left|3 p, m_{j}>\right|^{2}$ is the dipole transition matrix element between $3 p$ and $3 d$-states under the action of the dipole operator $C_{ \pm 1}^{(1)}\left(\mid 3 p, m_{j}>\right.$ : SO-split $p$-states; $\mid 3 d, m_{l}>$ : exchange-split $d$-states without SOC.) $\Delta$ is the resonance width of the $3 d$-states and $M_{\mathrm{S}}$ is the saturation magnetization.

XUV-single and double-pulse mSAXS experiments. The magnetic small-angle XUV scattering (mSAXS) experiments were conducted at the DiProl endstation [30] of the seeded free-electron laser FERMI, located at Elettra Sincrotrone Trieste, Italy, delivering close to transform-limited pulses which have a Gaussian intensity profile in time. For the experiments, the FEL source was lasing at the $12^{\text {th }}$ harmonic of the seed laser with a pulse duration of $230 \mathrm{fs}$, i.e., the FEL pulse length (FWHM) was $t_{\mathrm{FEL}}=$ $\frac{7}{6} \times\left(\frac{140 \mathrm{fs}}{\sqrt[3]{12}}\right)=71 \mathrm{fs}$ [62]. We employed the standard single-pulse and the recently developed double-pulse mode [31] setting the time delay between both FEL sub-pulses to $200 \mathrm{fs}$. For both modes, we used circularly polarized radiation allowing for the most stable operation of the FEL. For the single-pulse mode, the wavelength was set to $\lambda_{\mathrm{ph}, 1 \mathrm{P}}=20.3 \mathrm{~nm}$. In the double-pulse mode, the wavelengths were shifted away from the single-pulse wavelength by $\approx \pm 0.035 \mathrm{~nm}$. The wavelength separation in the double-pulse mode of $\Delta \lambda / \lambda \approx 0.2 \%$ is about twice the bandwidth (inset of Fig. 1(a)), allowing the determination of the energy of the sub-pulses on a (double-)shot-to-(double-)shot basis [63]. The mSAXS measurements were performed in transmission geometry and the scattered intensity was recorded using a CCD detector placed $50 \mathrm{~mm}$ behind the sample. Multi-shot measurements with exposure times of the CCD between $2 \mathrm{~s}$ and $50 \mathrm{~s}$ (20 to 500 FEL shots, respectively) were performed at low FEL fluences in the range from $0.3 \leq f \leq 15 \mathrm{~mJ} / \mathrm{cm}^{2}$ (non-destructive regime). Additionally, single-shot measurements were carried out for fluences $15<f \leq 43 \mathrm{~mJ} / \mathrm{cm}^{2}$ (destructive regime for $f \gtrsim$ $20 \mathrm{~mJ} / \mathrm{cm}^{2}$ ).

The spatial FEL-spot profile is composed of an intense center and broad tails that have been cut horizontally and vertically before the focusing optics of the beamline (bendable planar mirrors in Kirkpatrick-Baez (KB) configuration) by a set of beam-defining blades in front of the KB system, so that the beam profile at the sample position is almost 2D-Gaussian with an elliptical cross-section. The horizontal $\left(b_{x, \mathrm{FWHM}}\right)$ and vertical spot sizes $\left(b_{y, \mathrm{FWHM}}\right)$ at full width half maximum intensity $(\mathrm{FWHM})$ determine the peak fluence $f$ for a given pulse energy $E$ according to $f=a E /\left(b_{x, \mathrm{FWHM}} \cdot b_{y, \mathrm{FWHM}}\right)$ with $a=4 \ln 2 / \pi \approx 0.88$. A critical comment on the fluence definition in the case of a 2D-Gaussian beam profile is given in Supplement $D$.

The spot sizes $b_{x, \mathrm{FWHM}}$ and $b_{y \text {,FWHM }}$ at the sample position were determined from CCD images of the scintillation excited by the beam on a phosphorous screen. The spot sizes are determined to (70 \pm 
5) $\mathrm{x}(145 \pm 5) \mu \mathrm{m}^{2}$ for the multi-shot data and $(65 \pm 10) \times(130 \pm 10) \mu m^{2}$ for the single-shot data. Between single-pulse and double-pulse mode, the source point and hence the irradiated area at the sample position was slightly different of the order of $\pm 5 \%$ (corresponding to different spot sizes of about $\pm 2- \pm 3 \mu \mathrm{m}$, which was below the detection limit of our optical control of the beam profile) [31]. This difference was considered as a relative systematic error in the fluence determination between both single and double-pulse mode as mentioned in the text.

For the determination of the pulse energy $E_{\text {pulse }}$ at the sample, the measured value $E_{\mathrm{PADReS}}$ obtained by using PADReS' calibrated gas-detector intensity monitors [64] located at the beginning of the photontransport section, was propagated along the beamline. In order to take into account the effect of the above-mentioned spatial filtering in front of the KB system, the intensity of the incoming beam profile was measured on a scintillator screen with and without the beam-defining blades in the optical path. We estimate a loss of $30 \%(20 \%)$ in the pulse energy at the sample position for multi-shot mode (singleshot mode) due to the introduced spatial filtering. The transmission of the beamline optics is $70 \%$. In addition, an Al filter with a transmittance of $60 \%$ was used during the experiments to remove the UV seeding radiation $\left(\lambda_{\text {seed }} \approx 244 \mathrm{~nm}\right)$ from the XUV light produced by the FEL source. Finally, the transmittance of the $50 \mathrm{~nm}$ thick $\mathrm{Si}_{3} \mathrm{~N}_{4}$ membrane of $39 \%$ was considered. A variety of Al absorbers with calibrated transmittance $t_{\mathrm{abs}}$ were additionally used to vary the pulse energy and hence the fluence during the experiment. Taking into account all the above-mentioned attenuations, the pulse energy $E_{\text {pulse }}$ at the sample position is finally given by $E_{\text {pulse }}^{\text {single-shot }}=E_{\mathrm{PADReS}} \cdot 0.8 \cdot 0.7 \cdot 0.6 \cdot 0.39 \cdot t_{\mathrm{abs}}$ and $E_{\text {pulse }}^{\text {multi-shot }}=$ $E_{\mathrm{PADReS}} \cdot 0.7 \cdot 0.7 \cdot 0.6 \cdot 0.39 \cdot t_{\mathrm{abs}}$.

The accuracy of the spot-size determination results in an error for the irradiated area of about $\pm 20 \%$ for the single-shot mode. However, with the present fluence calibration the single-shot damage threshold of $\approx 20 \mathrm{~mJ} / \mathrm{cm}^{2}$ for Co M-edge radiation determined during the current experiment corresponds very well to the damage threshold determined during our previous experiments at FLASH and FERMI FELs on nominally identical Co/Pt samples using similar photon energies $[8,20]$. Hence, the absolute fluence scale is reliable within the above-mentioned accuracy of $\pm 20 \%$.

For the scattering experiments the beam was centered on a membrane (dimensions of $200 \times 200 \mu \mathrm{m}^{2}$ ), so that scattering from the membrane edges was minimized. Since the spot sizes were by a factor of $\approx 3$ (horizontal) and $\approx 1.5$ (vertical) smaller than the membrane size, one membrane was addressed at a time. The sample was mounted in such a way that the FEL beam impinged on the $\mathrm{Si}_{3} \mathrm{~N}_{4}$ membrane first before being scattered from the Co/Pt multilayer.

In the single-shot case (25-shot, i.e., multi-shot case), for a fluence of $43 \mathrm{~mJ} / \mathrm{cm}^{2}\left(7 \mathrm{~mJ} / \mathrm{cm}^{2}\right.$, see Fig. $1(\mathrm{c}))$, the number of photons per pixel in the maximum of the scattering ring is $\approx 0.42(\approx 3.6)$. Since the signal to noise ratio is $<0.05$ photons/pixel this intensity is sufficient to get smooth $I\left(Q=\sqrt{Q_{x}^{2}+Q_{y}^{2}}\right)$ curves (obtained from azimuthal averaging).

All data for the multi-shot experiments (fluences $f \leq 15 \mathrm{~mJ} / \mathrm{cm}^{2}$ ) were acquired using 3 individual membranes in total. In order to prove that there was no degradation of the structural and magnetic properties during the multi-shot experiments, we have checked that there is no dependence of the $Q$ position of the maximum scattering intensity $Q_{\max }$ on the FEL fluence. From all the multi-shot data, we determined an average value of $Q_{\max }=(0.036 \pm 0.001) \mathrm{nm}^{-1}$. For the whole fluence range $f \leq$ $15 \mathrm{~mJ} / \mathrm{cm}^{2}$, we can therefore rule out any structural damages (like intermixing of Co and $\mathrm{Pt}$ ) which had otherwise affected the intrinsic magnetic properties like magnetic anisotropy and/or saturation magnetization. Since both dictate the domain size $d=\pi / Q_{\max }$, a constant $Q_{\max }$ indicates structural integrity during the multi-shot experiments.

For the single-shot experiments, i.e., for fluences $f>15 \mathrm{~mJ} / \mathrm{cm}^{2}$, we used a fresh membrane after each shot. In total, 23 membranes were used ( 5 membranes for single-pulse and 18 membranes for doublepulse mode).

In the single-pulse mode, the pulse-intensity variation from shot to shot was around $20 \%$ (which is relevant only for the multi-shot mode).

In the double-pulse mode, typical intensity differences between both sub-pulses were $\left(I_{1}-I_{2}\right) /\left(I_{1}+\right.$ $\left.I_{2}\right)=0.0 \pm 0.4$. No correlation between asymmetry in intensity and scattering efficiency was observed for the single-shot data.

Data Analysis. The analysis of the scattering images, as shown in Fig. 1(b), was performed analog to Ref. [8]. The scattering images were azimuthally averaged after masking of the beamstop shadow and the charge-scattering streaks generated from the membrane edges. The resulting curves were fitted by 
$S(Q)=S\left(Q_{\text {peak }}\right)\left(1+\frac{\left(Q-Q_{\text {peak }}\right)^{2}}{\alpha_{1,2}^{2} \beta_{1,2}}\right)^{-\beta_{1,2}}+B_{0} Q^{-4}+C_{\mathrm{B}}$

where $C_{\mathrm{B}}$ accounts for a constant background and $B_{0} Q^{-4}$ describes a residual charge-scattering signal originating, e.g., from waviness of the membranes on the micrometer length scale due to strain after film growth, close to the center of the scattering image where the magnetic signal is small. The first term is the split Pearson type-VII distribution function that successfully describes the magnetic scattering signal as skewness and kurtosis are accounted for, originating from the domain-size distribution [64]. $S\left(Q_{\text {peak }}\right)$ is the intensity of the distribution function at the peak position $Q_{\text {peak }}$, and $\alpha_{1,2}^{2}$ and $\beta_{1,2}$ are parameters which have different values on the low and high- $Q$ side of the peak (indices 1 and 2 , respectively) reflecting the asymmetry of $S(Q)$.

\section{Data availability}

The data that support the findings of this study are available from the corresponding authors upon request. 


\section{References}

[1] F. Bencivenga, F. Capotondi, E. Principi, M. Kiskinova, C. Masciovecchio, Adv. Phys. 63, 327 (2015).

[2] E. A. Seddon, J. A. Clarke, D. J. Dunning, C. Masciovecchio, C. J. Milne, F. Parmigiani, D. Rugg, J. C. H. Spence, N. R. Thompson, K. Ueda, S. M. Vinko, J. S. Wark, W. Wurth, Rep. Prog. Phys. 80, 115901 (2017).

[3] E. Beaurepaire, J.-C. Merle, A. Daunois, J.-Y. Bigot, Phys. Rev. Lett. 76, 4250 (1996).

[4] B. Koopmans, G. Malinowski, F. Dalla Longa, D. Steiauf, M. Fahnle, T. Roth, M. Cinchetti, M. Aeschlimann, Nat. Mat. 9, 259 (2010).

[5] A. Kirilyuk, A. V. Kimel, T. Rasing, Rev. Mod. Phys. 82, 2731 (2010).

[6] D. Sander, S. O. Valenzuela, D. Makarov, C. H. Marrows, E. E. Fullerton, P. Fischer, J. McCord, P. Vavassori, S. Mangin, P. Pirro, B. Hillebrands, A. D. Kent, T. Jungwirth, O. Gutfleisch, C. G. Kim, A. Berger, J. Phys. D: Appl. Phys. 50, 363001 (2017).

[7] C. Dornes, Y. Acremann, M. Savoini, M. Kubli, M. J. Neugebauer, E. Abreu, L. Huber, G. Lantz, C. A. F. Vaz, H. Lemke, E. M. Bothschafter, M. Porer, V. Esposito, L. Rettig, M. Buzzi, A. Alberca, Y. W. Windsor, P. Beaud, U. Staub, Diling Zhu, Sanghoon Song, J. M. Glownia, S. L. Johnson, Nature 565, 209 (2019).

[8] B. Pfau, S. Schaffert, L. Müller, C. Gutt, A. Al-Shemmary, F. Büttner, R. Delaunay, S. Düsterer, S. Flewett, R. Frömter, J. Geilhufe, E. Guehrs, C. M. Günther, R. Hawaldar, M. Hille, N. Jaouen, A. Kobs, K. Li, J. Mohanty, H. Redlin, W. F. Schlotter, D. Stickler, R. Treusch, B. Vodungbo, M. Kläui, H. P. Oepen, J. Lüning, G. Grübel, S. Eisebitt, Nat. Commun. 3, 1100 (2012).

[9] B. Vodungbo, J. Gautier, G. Lambert, A. Barszczak Sardinha, M. Lozano, S. Sebban, M. Ducousso, W. Boutu, K. Li, B. Tudu, M. Tortarolo, R. Hawaldar, R. Delaunay, V. López-Flores, J. Arabski, C. Boeglin, H. Merdji, P. Zeitoun, J. Lüning, Nat. Commun. 3, 999 (2012).

[10] C. E. Graves et al., Nat. Mater. 12, 293 (2013).

[11] M. Malvestuto, R. Ciprian, A. Caretta, B. Casarin, F. Parmigiani, J. Phys. Cond. Mat. 30, 053002 (2018).

[12] B. Nagler, U. Zastrau, R. R. Fäustlin, S. M. Vinko, T. Whitcher, a. J. Nelson, R. Sobierajski, J. Krzywinski, J. Chalupsky, E. Abreu, S. Bajt, T. Bornath, T. Burian, H. Chapman, J. Cihelka, T. Döppner, S. Düsterer, T. Dzelzainis, M. Fajardo, E. Förster, C. Fortmann, E. Galtier, S. H. Glenzer, S. Göde, G. Gregori, V. Hajkova, P. Heimann, L. Juha, M. Jurek, F. Y. Khattak, A. R. Khorsand, D. Klinger, M. Kozlova, T. Laarmann, H. J. Lee, R. W. Lee, K.-H. Meiwes-Broer, P. Mercere, W. J. Murphy, A. Przystawik, R. Redmer, H. Reinholz, D. Riley, G. Röpke, F. Rosmej, K. Saksl, R. Schott, R. Thiele, J. Tiggesbäumker, S. Toleikis, T. Tschentscher, I. Uschmann, H. J. Vollmer, J. S. Wark, Nat. Phys. 5, 693 (2009).

[13] L. Young, E. P. Kanter, B. Krässig, Y. Li, a M. March, S. T. Pratt, R. Santra, S. H. Southworth, N. Rohringer, L. F. Dimauro, G. Doumy, C. a Roedig, N. Berrah, L. Fang, M. Hoener, P. H. Bucksbaum, J. P. Cryan, S. Ghimire, J. M. Glownia, D. a Reis, J. D. Bozek, C. Bostedt, M. Messerschmidt, Nature 466, 56 (2010).

[14] S. M. Vinko, O. Ciricosta, B. I. Cho, K. Engelhorn, H.-K. Chung, C. R. D. Brown, T. Burian, J. Chalupský, R. W. Falcone, C. Graves, V. Hájková, A. Higginbotham, L. Juha, J. Krzywinski, H. J. Lee, M. Messerschmidt, C. D. Murphy, Y. Ping, A. Scherz, W. Schlotter, S. Toleikis, J. J. Turner, L. Vysin, T. Wang, B. Wu, U. Zastrau, D. Zhu, R. W. Lee, P. A. Heimann, B. Nagler, J. S. Wark, Nature 482, 59 (2012). 
[15] N. Rohringer, D. Ryan, R. A. London, M. Purvis, F. Albert, J. Dunn, J. D. Bozek, C. Bostedt, A. Graf, R. Hill, S. P. Hau-Riege, J. J. Rocca, Nature 481, 488 (2012).

[16] B. Zaks, R. B. Liu, M. S. Sherwin, Nature 483, 580 (2012).

[17] M. Beye, S. Schreck, F. Sorgenfrei, C. Trabant, N. Pontius, C. Schüßler-Langeheine, W. Wurth, A. Föhlisch, Nature 501, 191 (2013).

[18] H. Yoneda, Y. Inubushi, K. Nagamine, Y. Michine, H. Ohashi, H. Yumoto, K. Yamauchi, H. Mimura, H. Kitamura, T. Katayama, T. Ishikawa, M. Yabashi, Nature 524, 446 (2015).

[19] H. Yoneda, Y. Inubushi, M. Yabashi, T. Katayama, T. Ishikawa, H. Ohashi, H. Yumoto, K. Yamauchi, H. Mimura, H. Kitamura, Nat. Commun. 5, 5080 (2014).

[20] L. Müller, C. Gutt, B. Pfau, S. Schaffert, J. Geilhufe, F. Büttner, J. Mohanty, S. Flewett, R. Treusch, S. Düsterer, H. Redlin, A. Al-Shemmary, M. Hille, A. Kobs, R. Frömter, H. P. Oepen, B. Ziaja, N. Medvedev, S.-K. Son, R. Thiele, R. Santra, B. Vodungbo, J. Lüning, S. Eisebitt, G. Grübel, Phys. Rev. Lett. 110, 234801 (2013).

[21] B. Wu, T. Wang, C. E. Graves, D. Zhu, W. F. Schlotter, J. J. Turner, O. Hellwig, Z. Chen, H. A. Dürr, A. Scherz, J. Stöhr, Phys. Rev. Lett. 117, 027401 (2016).

[22] Z. Chen, D. J. Higley, M. Beye, M. Hantschmann, V. Mehta, O. Hellwig, A. Mitra, S. Bonetti, M. Bucher, S. Carron, T. Chase, E. Jal, R. Kukreja, T. Liu, A. H. Reid, G. L. Dakovski, A. Föhlisch, W. F. Schlotter, H. A. Dürr, J. Stöhr, Phys. Rev. Lett. 121, 137403 (2018).

[23] J. Stöhr and A. Scherz, Phys. Rev. Lett. 115, 107402 (2015).

[24] The XMCD effect at $K$ edges is comparably small for $3 d$ metals, hence, mSAXS experiments are challenging. Besides, rare-earth elements exhibiting $L$ edges in the hard $X-$ ray regime were not studied so far.

[25] M. Schneider, B. Pfau, C. M. Günther, C. von Korff Schmising, D. Weder, J. Geilhufe, J. Perron, F. Capotondi, E. Pedersoli, M. Manfredda, M. Hennecke, B. Vodungbo, J. Lüning, S. Eisebitt, Phys. Rev. Lett. 125, 127201 (2020).

[26] D. J. Higley, A. H. Reid, Z. Chen, L. Le Guyader, O. Hellwig, A. A. Lutman, T. Liu, P. Shafer, T. Chase, G. L. Dakovski, A. Mitra, E. Yuan, J. Schlappa, H. A. Dürr, W. F. Schlotter, J. Stöhr, Nat. Commun. 10, 5289 (2019).

[27] Indications for an onset of quenching at $f \approx 10 \mathrm{~mJ} / \mathrm{cm}^{2}$ for $L_{3}$ edge radiation were reported in Ref. [28] for the magnetic scattering of a Co/Pd multilayer using Fourier-transform holography technique and it was speculated that the quenching is a consequence of X-ray radiation-induced demagnetization.

[28] T. Wang, D. Zhu, B. Wu, C. Graves, S. Schaffert, T. Rander, L. Müller, B. Vodungbo, C. Baumier, D. P. Bernstein, B. Bräuer, V. Cros, S. de Jong, R. Delaunay, A. Fognini, R. Kukreja, S. Lee, V. López-Flores, J. Mohanty, B. Pfau, H. Popescu, M. Sacchi, A. B. Sardinha, F. Sirotti, P. Zeitoun, M. Messerschmidt, J. J. Turner, W. F. Schlotter, O. Hellwig, R. Mattana, N. Jaouen, F. Fortuna, Y. Acremann, C. Gutt, H. A. Dürr, E. Beaurepaire, C. Boeglin, S. Eisebitt, G. Grübel, J. Lüning, J. Stöhr, A. O. Scherz, Phys. Rev. Lett. 108, 267403 (2012).

[29] G. Winkler, A. Kobs, A. Chuvilin, D. Lott, A. Schreyer, H. P. Oepen, J. Appl. Phys. 117, 105306 (2015).

[30] E. Pedersoli, F. Capotondi, D. Cocco, M. Zangrando, B. Kaulich, R. H. Menk, A. Locatelli, T. O. Mentes, C. Spezzani, G. Sandrin, D. M. Bacescu, M. Kiskinova, S. Bajt, M. Barthelmess, A. Barty, J. Schulz, L. Gumprecht, H. N. Chapman, A. J. Nelson, M. Frank, M. J. Pivovaroff, B. W. Woods, M. J. Bogan, and J. Hajdu, Rev. Sci. Instr. 82, 043711 (2011). F. Capotondi, E. Pedersoli, N. Mahne, R. H. Menk, G. Passos, L. Raimondi, C. Svetina, G. Sandrin, M. Zangrando, M. Kiskinova, S. Bajt, M. Barthelmess, H. Fleckenstein, H. N. Chapman, J. Schulz, 
J. Bach, R. Frömter, S. Schleitzer, L. Müller, C. Gutt, G. Grübel, Rev. Sci. Instr. 84, 051301 (2013).

[31] E. Allaria, F. Bencivenga, R. Borghes, F. Capotondi, D. Castronovo, P. Charalambous, P. Cinquegrana,M.B. Danailov, G. De Ninno, A. Demidovich, S. Di Mitri, B. Diviacco, D. Fausti, W.M. Fawley, E. Ferrari, L. Froehlich, D. Gauthier, A. Gessini, L. Giannessi, R. Ivanov, M. Kiskinova, G. Kurdi, B. Mahieu, N. Mahne, I. Nikolov, C. Masciovecchio, E. Pedersoli, G. Penco, L. Raimondi, C. Serpico, P. Sigalotti, S. Spampinati, C. Spezzani, C. Svetina, M. Trovò, M. Zangrando, Nat. Commun. 4, 2476 (2013).

[32] G. De Ninno, B. Mahieu, E. Allaria, L. Giannessi, S. Spampinati, Phys. Rev. Lett. 110, 064801 (2013), B. Mahieu, E. Allaria, D. Castronovo, M. B. Danailov, A. Demidovich, G. De Ninno, S. Di Mitri, W. M. Fawley, E. Ferrari, L. Fröhlich, D. Gauthier, L. Giannessi, N. Mahne, G. Penco, L. Raimondi, S. Spampinati, C. Spezzani, C. Svetina, M. Trovò, M. Zangrando, Opt. Express 21, 22728 (2013).

[33] S. Valencia, A. Gaupp, W. Gudat, H.-Ch. Mertins, P. M. Oppeneer, D. Abramsohn, C. M. Schneider, N. J. Phys. 8, 254 (2006).

[34] J. Stöhr and H. C. Siegmann, Magnetism: From Fundamentals to Nanoscale Dynamics, (Springer, New York, 2006).

[35] B. Ziaja et al., EPJD 40, 465 (2006), B. Ziaja, H. Wabnitz, E. Weckert, T. Moeller, New J. Phys. 10, 043003 (2008), B. Ziaja, H. Wabnitz, E. Weckert, T. Moeller, Euro. Phys. Lett. 82, 24002 (2008), F. Wang, E. Weckert, B. Ziaja, J. Plasma Phys. 75, 289 (2009), B. Ziaja, T. Laarmann, H. Wabnitz, F. Wang, E. Weckert, C. Bostedt, T. Moeller, New J. Phys 11, 103012 (2009). B. Ziaja, H. Wabnitz, F. Wang, E. Weckert, T. Moeller, Phys. Rev. Lett. 102, 205002 (2009).

[36] T. Roth, A. J. Schellekens, S. Alebrand, O. Schmitt, D. Steil, B. Koopmans, M. Cinchetti, M. Aeschlimann, Phys. Rev. X 2, 021006 (2012).

[37] N. Bergeard, M. Hehn, S. Mangin, G. Lengaigne, F. Montaigne, M. L. M. Lalieu, B. Koopmans, G. Malinowski, Phys. Rev. Lett. 117, 147203 (2016).

[38] I. Radu, C. Stamm, A. Eschenlohr, F. Radu, R. Abrudan, K. Vahaplar, T. Kachel, N. Pontius, R. Mitzner, K. Holldack, A. Föhlisch, T. A. Ostler, J. H. Mentink, R. F. L. Evans, R. W. Chantrell, A. Tsukamoto, A. Itoh, A. Kirilyuk, A. V. Kimel, Th. Rasing, SPIN 5, 1550004 (2015).

[39] N. Moisan, G. Malinowski, J. Mauchain, M. Hehn, B. Vodungbo, J. Lüning, S. Mangin, E. E. Fullerton, A. Thiaville, Sci. Rep. 4, 4658 (2014).

[40] K. C. Kuiper, T. Roth, A. J. Schellekens, O. Schmitt, B. Koopmans, M. Cinchetti, M. Aeschlimann, Appl. Phys. Lett. 105, 202402 (2014).

[41] S.-g. Gang, R. Adam, M. Plötzing, M. von Witzleben, C. Weier, U. Parlak, D. E. Bürgler, C. M. Schneider, Phys. Rev. B 97, 064412 (2018).

[42] J. Chen, U. Bovensiepen, A. Eschenlohr, T. Müller, P. Elliott, E. K. U. Gross, J. K. Dewhurst, S. Sharma, Phys. Rev. Lett. 122, 067202 (2019).

[43] F. Siegrist, J. A. Gessner, M. Ossiander, C. Denker, Y.-P. Chang, M. C. Schröder, A. Guggenmos, Y. Cui, J. Walowski, U. Martens, J. K. Dewhurst, U. Kleineberg, M. Münzenberg, S. Sharma, M. Schultze, Nature 571, 240 (2019).

[44] B. Koopmans, J. J. M. Ruigrok, F. Dalla Longa, W. J. M. de Jonge, Phys. Rev. Lett. 95, 267207 (2005). B. Koopmans, H. H. J. E. Kicken, M. van Kampen, W. J. M. de Jonge, J. Magn. Magn. Mat. 286, 271 (2005). 
[45] M. Battiato, K. Carva, P. M. Oppeneer, Phys. Rev. Lett. 105, 027203 (2010), K. Carva, M. Battiato, P. M. Oppeneer, Phys. Rev. Lett. 107, 207201 (2011), M. Battiato, K. Carva, P. M. Oppeneer, Phys. Rev. B 86, 024404 (2012).

[46] R. Allenspach, D. Mauri, M. Taborelli, M. Landolt, Phys. Rev. B 35, 4801 (1987).

[47] L. E. Klebanoff, D. G. Van Campen, R. J. Pouliot, Phys. Rev. B 49, 2047 (1994).

[48] M. Bauer, A. Marienfeld, M. Aeschlimann, Prog. Surf. Sci. 90, 319 (2015).

[49] M. Krauß, T. Roth, S. Alebrand, D. Steil, M. Cinchetti, M. Aeschlimann, H. C. Schneider, Phys. Rev. B 80, 180407(R) (2009).

[50] D. Steiauf and M. Fähnle, Phys. Rev. B 79,140401(R) (2009).

[51] M. P. Seah, W. A. Dench, Surf. Interface Anal. 1, 1 (1979).

[52] M. Aeschlimann, M. Bauer, S. Pawlik, W. Weber, R. Burgermeister, D. Oberli, H. C. Siegmann, Phys. Rev. Lett. 79, 5158 (1997).

[53] A. Goris, K. M. Döbrich, I. Panzer, A. B. Schmidt, M. Donath, M. Weinelt, Phys. Rev. Lett. 107, 026601 (2011).

[54] C. Chen, Z. Tao, A. Carr, P. Matyba, T. Szilvási, S. Emmerich, M. Piecuch, M. Keller, D. Zusin, S. Eich, M. Rollinger, W. You, S. Mathias, U. Thumm, M. Mavrikakis, M. Aeschlimann, P. M. Oppeneer, H. Kapteyn, M. Murnane, Proc. Nat. Acad. Sci. 114, E5300 (2017).

[55] P. B. Allen, Phys. Rev. Lett. 59, 1460 (1987).

[56] L. Waldecker, R. Bertoni, R. Ernstorfer, J. Vorberger, Phys. Rev. X 6, 021003 (2016).

[57] S. T. Weber and B. Rethfeld, Appl. Surf. Sci. 417, 64 (2017).

[58] P. B. Johnson and R. W. Christy, Phys. Rev. B 9, 5056 (1974).

[59] U. Bierbrauer, S. T. Weber, D. Schummer, M. Barkowski, A.-K. Mahro, S. Mathias, H. C. Schneider, B. Stadtmüller, M. Aeschlimann, B. Rethfeld, J. Phys. Cond. Mat. 29, 244002 (2017).

[60] K. Bobowski, M. Gleich, N. Pontius, C. Schüßler-Langeheine, C. Trabant, M. Wietstruk, B. Frietsch, M. Weinelt, J. Phys. Cond. Mat. 29, 234003 (2017).

[61] C. Gutt, S. Streit-Nierobisch, L. M. Stadler, B. Pfau, C. M. Günther, R. Konnecke, R. Frömter, A. Kobs, D. Stickler, H. P. Oepen, R. R. Faustlin, R. Treusch, J. Feldhaus, E. Weckert, I. A. Vartanyants, M. Grunze, A. Rosenhahn, T. Wilhein, S. Eisebitt, G. Grübel, Phys. Rev. B 81, 100401 (2010).

[62] P. Finetti et al., Phys. Rev. X 7,021043 (2017).

[63] M. Zangrando, D. Cocco, C. Fava, S. Gerusina, R. Gobessi, N. Mahne, E. Mazzucco, L. Raimondi, L. Rumiz, C. Svetina, J. Synchrotron Rad. 22, 565 (2015).

[64] K. Bagschik, R. Frömter, J. Bach, B. Beyersdorff, L. Müller, S. Schleitzer, M. H. Berntsen, C. Weier, R. Adam, J. Viefhaus, C. M. Schneider, G. Grübel, H. P. Oepen, Phys. Rev. B 94, 134413 (2016).

\section{Acknowledgements}

The experimental study was carried out at the free-electron laser FERMI at Elettra and we would like to thank the scientific and technical teams for the support during the experiment. We thank Marco Battiato and Lars Bocklage for valuable discussions. A. P.-K., L. M., M. R., W. R., G. G. acknowledge funding by the Deutsche Forschungsgemeinschaft (DFG, German Research Foundation) - SFB-925 - project 170620586. K. B., J. W., R. F., H. P. O. acknowledge funding by DFG via SFB 668. R. F., H. P. O., and 
G. G. acknowledge support by the Cluster of Excellence "The Hamburg Centre of Ultrafast Imaging" of the DFG (EXC 1074 - project ID 194651731). M. H. B. acknowledges financial support by the Knut and Alice Wallenberg Foundation (Grant No. KAW 2012.0361). M. S. acknowledges support from the INTER-EXCELLENCE program of the Ministry of Education, Youth, and Sports of the Czech Republic (Grant No. LTT 17015).

\section{Author contributions}

L.M., M.H.B., W.R., K.B., J.W., R.F. conceived the experiment. M.D., F.C., E.P., M.M., M.K. operated the FEL beamline. A.P.-K., K.B., R.F., and H.P.O. designed, produced and characterized the samples. L.M., M.H.B., W.R., K.B., J.W., R.F. carried out the mSAXS experiment. A.P.-K., L.M., and M.H.B. analyzed and interpreted the experimental results. L.M. and A.P.-K. developed and performed modeling in terms of demagnetization. A.S. conducted the calculations on stimulated emission. M.S., V.L., B.Z. conducted the Boltzmann calculations. A.P.-K. wrote the manuscript with discussion and improvements from all authors. G.G. supervised this work.

\section{Competing Interests}

The authors declare no competing interests.

\section{Additional Information}

Supplementary information The online version contains supplementary material available at XXX

Correspondence and request for materials should be addressed to A.P.-K.

\section{Peer review information}

Reprints and permission information is available at $X X$

Publisher's note Springer Nature remains neutral with regard to jurisdictional claims in published maps and institutional affiliations. 

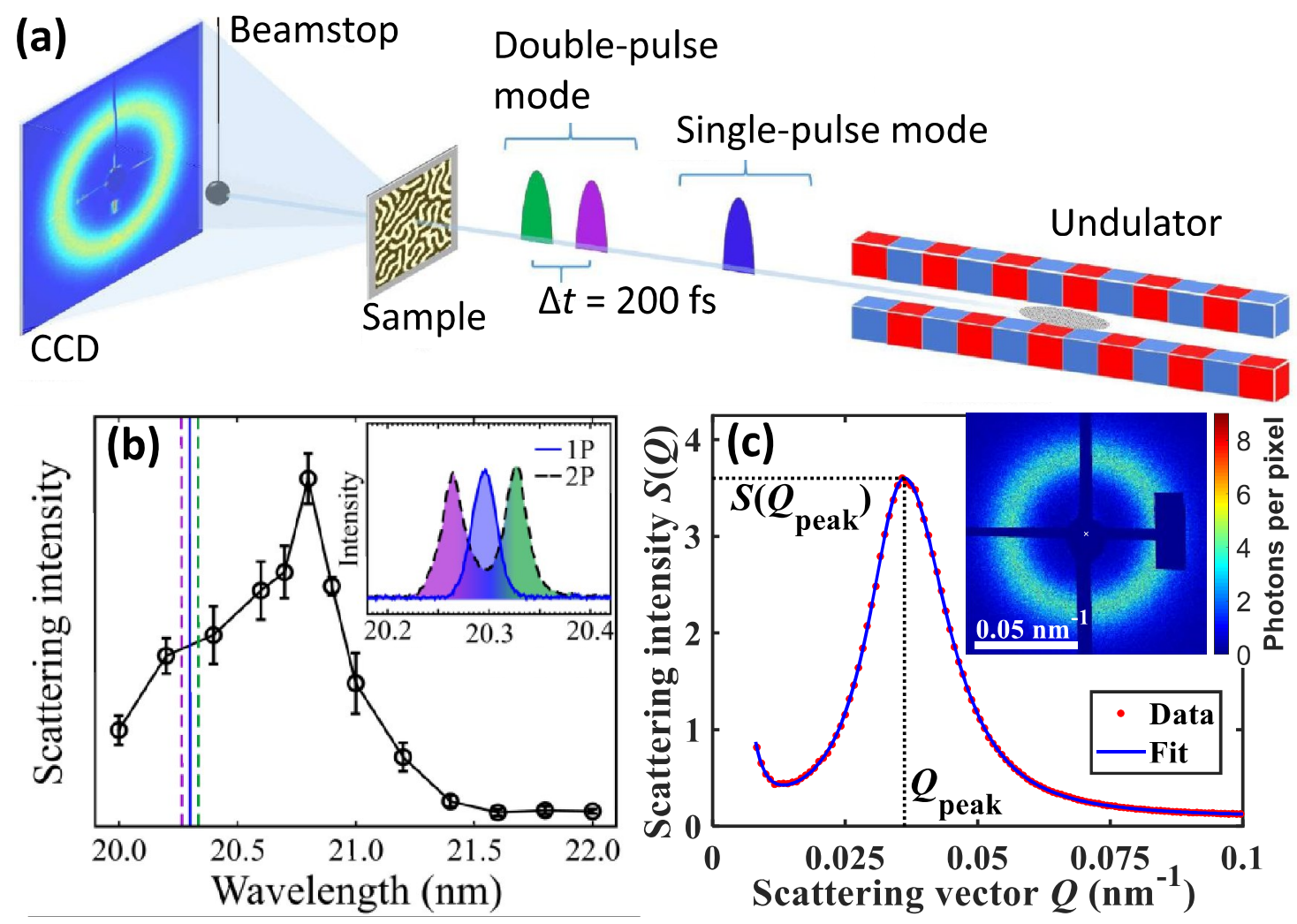

Fig. 1 mSAXS experiment and data analysis. (a) The magnetic multilayer with mazedomain pattern is either hit by a single or double FEL pulse and the mSAXS pattern is recorded by a CCD detector. (b) Scattering intensity vs wavelength covering the Co $M_{2,3}$ edge $\left(f \leq 3 \mathrm{~mJ} / \mathrm{cm}^{2}\right.$, linear-response regime). The vertical lines mark the wavelengths used for the $I_{\text {eff }}(f)$ experiments (blue: single-pulse, purple and green: double-pulse mode). The inset shows corresponding spectra for individual pulses. (c) Azimuthally averaged intensity vs scattering vector of the scattering image given as inset $\left(f=7 \mathrm{~mJ} / \mathrm{cm}^{2}\right)$. The solid line is a phenomenological fit (see Methods). 


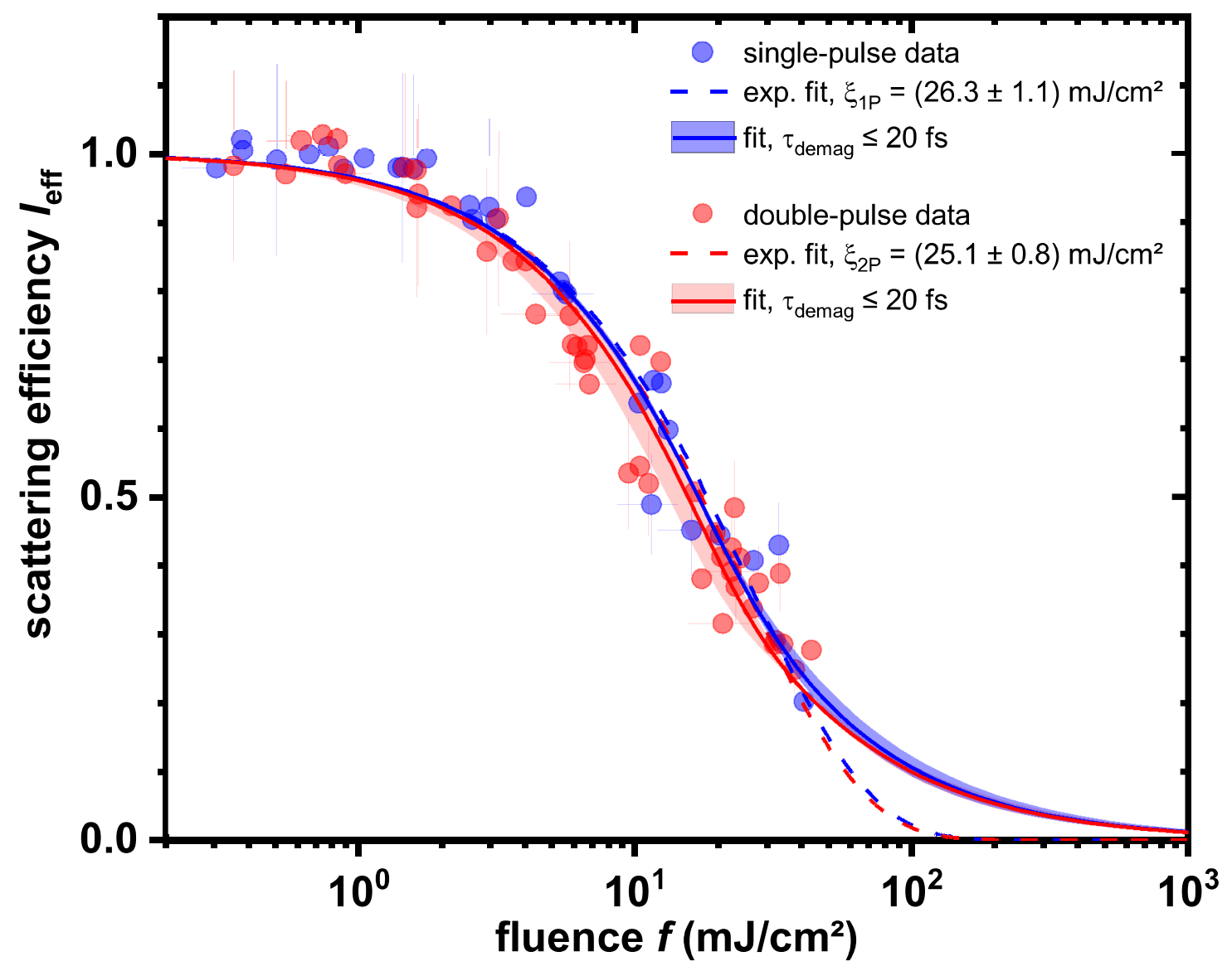

Fig. 2 Quenching of magnetic scattering. Scattering efficiency vs fluence for single-pulse (blue) and double-pulse data (red). Dashed lines are single-parameter exponential decay fits $\left(I_{\text {eff }}(f)=\exp (-f / \xi)\right)$. Solid lines are model curves based on the phenomenological description of demagnetization that are simultaneously fitted to single and double-pulse data. The shaded areas show the uncertainty of the modeling. 
low fluence regime high fluençe regime
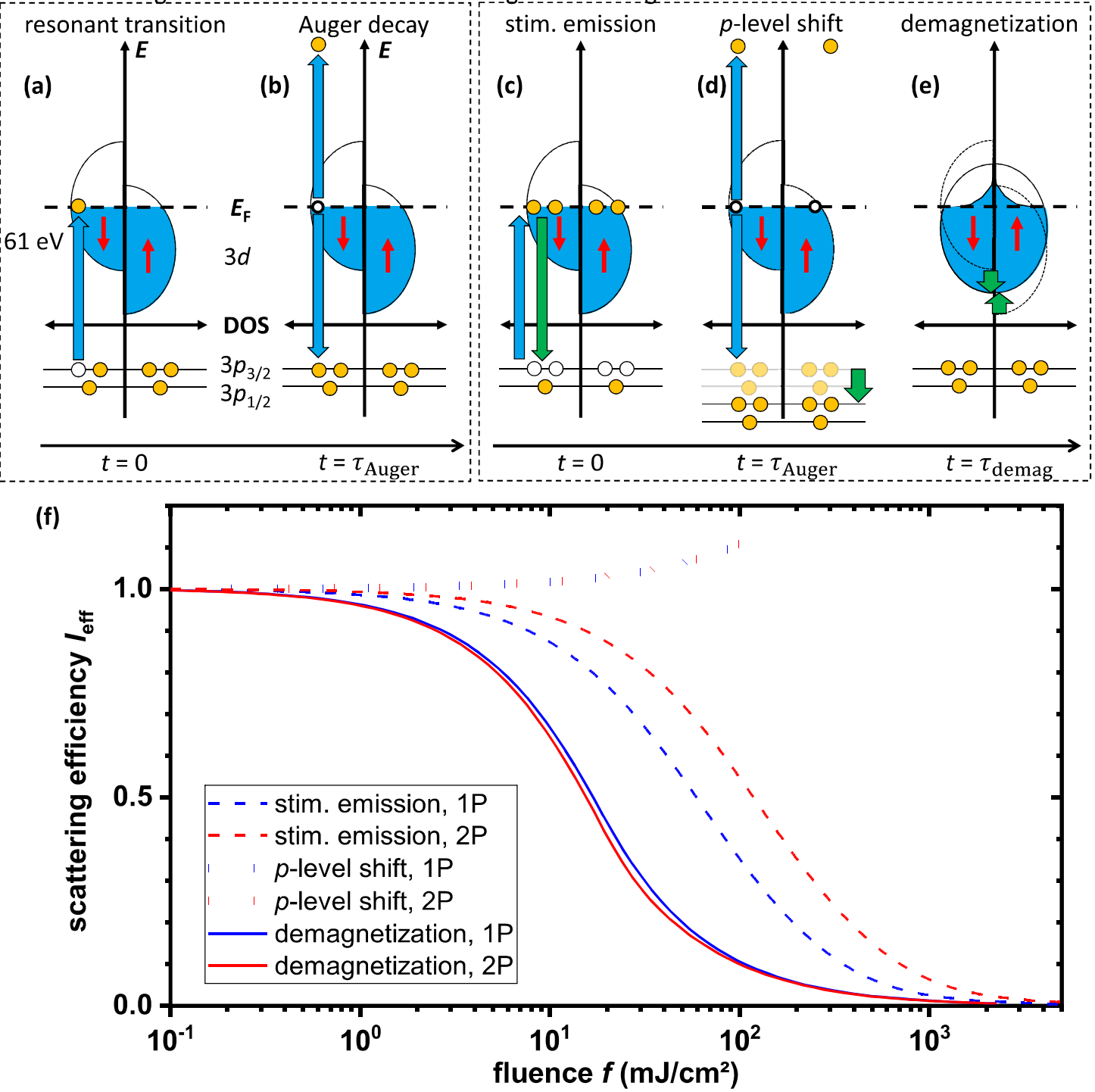

Fig. 3 Transient states and their impact on scattering efficiency. (a)-(e) Schematics for transient band structure at low (a)-(b) and high fluences (c)-(e). Yellow/white dots depict occupied/unoccupied electronic states; green arrows highlight modifications of electronic transitions and band structure at high fluences. (f) Corresponding predictions for the scattering efficiency $I_{e f f}(f)$ using single (1P,blue) and double-pulse schemes (2P,red) for stimulated emission (dashed lines), electron-environment-induced 3p-level shift (dotted lines), and ultrafast demagnetization (solid lines). 
(a)

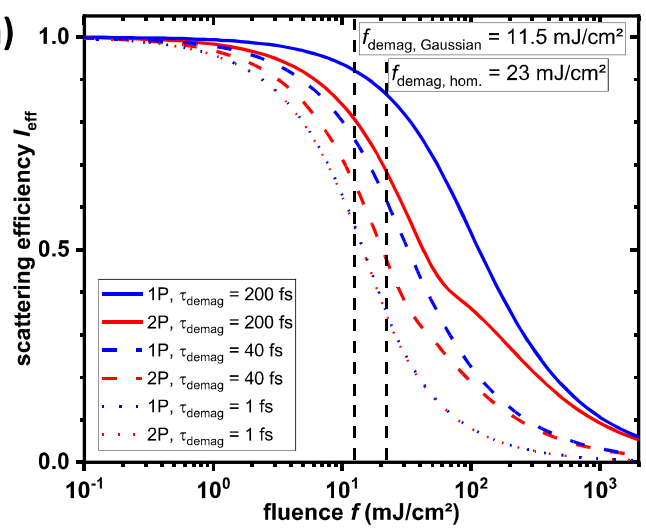

(b)

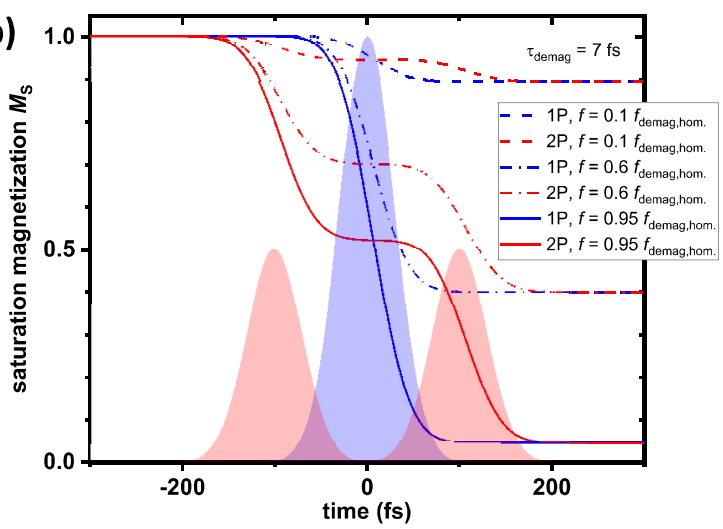

Fig. 4 Modeling of demagnetization for single-pulse (1P) and double-pulse (2P) schemes. (a) Scattering efficiency vs fluence $I_{\text {eff }}(f)$ for different demagnetization times $\tau_{\text {demag. }} f_{\text {demag,Gaussian }}$ and $f_{\text {demag,hom. }}$ correspond to the fluence for complete demagnetization in the spatial pulse center for a lateral Gaussian and a flat-top beam profile, respectively. (b) Temporal Gaussian pulse profiles (filled areas) and magnetization transients (lines) for a demagnetization time of $\tau_{\text {demag }}=7$ fs obtained from the best fit of the experimental data shown in Fig. 2. The temporal evolution of the saturation magnetization is shown for three different fluences as labeled. 


\section{Supplementary Files}

This is a list of supplementary files associated with this preprint. Click to download.

- SupplementNatureCommun210921final.pdf 